\title{
PROPOSTA DE IMPLANTAÇÃO DE UMA CENTRAL DE TRIAGEM DE RESÍDUOS SÓLIDOS EM CAMPINA GRANDE-PB
}

\section{PROPOSAL FOR THE IMPLEMENTATION OF A SOLID WASTE SCREENING CENTER IN CAMPINA GRANDE-PB}

VANESSA ROSALES BEZERRA, M.SC. | UEPB

LUIS REYES ROSALES MONTERO, Dr. | UFCG

VALDERI DUARTE LEITE, Dr. | UEPB

ADRIANO OLIVEIRA DA SILVA, |UEPB

YOHANNA JAMILA VILAR DE BRITO, M.SC.| UEPB

CARLOS ANTÔNIO PEREIRA DE LIMA, Dr. | UEPB

\begin{abstract}
RESUMO
As centrais de triagem têm um papel importante no processo de reciclagem de resíduos sólidos uma vez que viabilizam a separação dos diferentes tipos de materiais que compõem os resíduos para que os mesmos possam ser reutilizados ou transformados em novos produtos. Atualmente, a cidade de Campina Grande não dispõe de um programa de reciclagem e reutilização de resíduos para todo município, mesmo sendo obrigatório segundo as diretrizes da Política Nacional de Resíduos Sólidos. O objetivo deste trabalho é apresentar uma proposta para implantação central de triagem na cidade que realize a segregação de todos os resíduos gerados, para que as etapas de reutilização e reciclagem sejam viabilizadas. A central de triagem proposta para a cidade de Campina Grande-PB é do tipo semiautomática com o uso de esteiras e catadores para auxiliar na segregação dos resíduos. O presente manual apresenta especificações sobre os detalhes construtivos, organização do galpão e cotação dos equipamentos. Por fim, este artigo busca contribuir como uma referência em termos de critérios a serem analisados no processo de implantação e operação de triagem de resíduos sólidos urbanos
\end{abstract}

PALAVRAS-CHAVE: resíduos sólidos, triagem, reciclagem

\begin{abstract}
The sorting plants play an important role in the solid waste recycling process since they make it possible to separate the different types of materials that make up the waste so that it can be reused or transformed into new products. Currently, the city of Campina Grande does not have a waste recycling and reuse program for the entire municipality, even though it is mandatory according to the guidelines of the National Solid Waste Policy. The objective of this work is to present a proposal for a central sorting plant in the city that segregates all the waste generated, so that the stages of reuse and recycling are made possible. The sorting center proposed for the city of Campina Grande-PB is of the semi-automatic type with the use of mats and scavengers to assist in the segregation of waste. This manual presents specifications on the construction details, shed organization and equipment quotation. Finally, this article seeks to contribute as a reference in terms of criteria to be analyzed in the process of implantation and operation of sorting urban solid waste.
\end{abstract}

KEYWORD: solid waste, sorting, recycling 


\section{INTRODUÇÃO}

O atual modelo de produção e consumo imposto pela sociedade capitalista traz grandes preocupações relacionadas ao meio ambiente. Entre estas preocupações, a geração de resíduos, uma vez que o não tratamento dos resíduos sólidos pode causar sérios impactos socioambientais negativos(SOARES e PEREIRA,2017).

Segundo Martins et al., (2016) os resíduos sólidos, que antes eram vistos como subproduto de atividades humanas $\mathrm{e}$ industriais, ganharam maior importância com seu conceito agregado à recuperação dos materiais que deixam de ser considerados inservíveis. A redução da quantidade de resíduos depositada no solo é uma situação de emergência.

Para que qualquer tratamento de resíduos sólidos tenha êxito, é necessário separar o mesmo, considerando suas características físico-químicas. Quanto mais bem separado esses resíduos, maior o seu valor agregado (MACHADO, 2013).

Nesse contexto, as centrais de triagem têm um papel importante no processo de reciclagem de resíduos sólidos uma vez que viabilizam a separação dos diferentes tipos de materiais que compõem os resíduos para que os mesmos possam ser reutilizados ou transformados em novos produtos.

Os atuais hábitos consumistas da crescente população nos últimos anos têm gerado uma preocupação mundial em relação ao aumento exponencial da geração de resíduos sólidos que, associado a um sistema de gerenciamento inadequado, pode causar problemas ambientais, sociais e de saúde pública (ELK, 2007).

Assim, o equacionamento da relação geração-disposição de resíduos tornou-se uma meta desafiadora para gestores (JACOBI e BESEN, 2011). Para Seidel (2010), a grande produção de resíduos gera questionamentos sobre o que fazer, como tratar todo esse material e qual a contribuição desses esforços para as mudanças ambientais globais.

No Brasil, em 2018, foram geradas 79 milhões de toneladas de resíduos sólidos urbanos, um aumento de pouco menos de $1 \%$ em relação ao ano anterior, desse montante, 92\% (72,7 milhões) foram coletados, uma alta de 1,66\% em comparação a 2017 (ABELPRE, 2018). Essa discrepância de valores ilustra falhas existentes no processo técnico-operacional de gerenciamento dos resíduos sólidos no país.

Com base no presente cenário em que se encontra a problemática dos resíduos sólidos no Brasil, a Lei no 12.305/10 instituiu a Política Nacional de Resíduos Sólidos, que detêm instrumentos importantes para permitir o avanço necessário ao País no enfrentamento dos principais problemas ambientais, sociais e econômicos decorrentes do manejo inadequado dos resíduos sólidos
(BRASIL, 2010).

Nesse sentido, avanços na PNRS são necessários para contribuir na redução da geração de resíduos, assim como para diminuir custos de tratamento e riscos de contaminação no meio ambiente, contribuindo para a eficiência das diferentes etapas subsequentes, principalmente nas associações de materiais recicláveis (VITOR et al., 2015).

Oliveira e Pasqual (1998) afirmam que o gerenciamento de resíduos sólidos de forma integrada é constituído por ações normativas, operacionais, financeiras e de planejamento e que, para ser eficaz, deve contemplar o uso de práticas administrativas de resíduos, com manejo seguro e efetivo fluxo de RSU, com o mínimo de impactos sobre a saúde pública e o meio ambiente.

O componente operacional da gestão de resíduos sólidos inclui as etapas de segregação, coleta, transporte, tratamento e disposição final. A implementação de tais etapas nos municípios ou consórcio de municípios tornou-se obrigatória após a instituição da PNRS (BRASIL, 2010).

Segundo Martinho \& Gonçalves (2000), centrais de triagem são unidades nas quais os resíduos misturados, ou previamente separados na origem, são triados e processados mecânica e/ou manualmente, com o objetivo de recuperar diferentes fileiras para reciclagem e/ou determinados fluxos para subsequente processamento e valoração.

A princípio, este trabalho disserta sobre os temas de resíduos sólidos, triagem e reciclagem com o intuito de embasar o leitor acerca da importância de uma central de triagem no gerenciamento de resíduos sólidos. Em seguida, são expostos detalhes e recomendações técnicas sobre a central de triagem proposta nesse manual.

Dessa forma, com base nas diretrizes da Política Nacional de Resíduos Sólidos (PNRS), este artigo visa apresentar uma proposta de Central de Triagem de Resíduos Sólidos para ser implantada na cidade de Campina Grande-PB.

\section{METODOLOGIA}

Atualmente, o sistema de gerenciamento de resíduos em Campina Grande-PB, conta com as etapas de coleta (através de caminhões regulares e/ou compactadores) e disposição final por meio do Aterro Sanitário de Campina Grande, localizado em Catolé de Boa Vista, zona rural do município de Campina Grande-PB.

Entretanto, a referida cidade não dispõe de um programa de reciclagem e reutilização de resíduos, mesmo sendo obrigatório segundo as diretrizes da PNRS. Com isso, é necessário a implantação de uma central de triagem na cidade para que as etapas de reutilização e reciclagem sejam viabilizadas. 
Segundo a norma NBR 10004/04, resíduos sólidos são substâncias nos estados sólido e semissólido, que resultam de atividades de origem industrial, doméstica, hospitalar, comercial, agrícola, de serviços e de varrição.

As estações de triagem possibilitam uma redução da quantidade de resíduos a serem dispostos em aterros sanitários (como resultado de uma melhor recuperação dos materiais recicláveis), além de favorecer a qualidade dos materiais recuperados, tornando-os mais competitivos em termos de mercado (MARTINHO e GONÇALVES, 2000).

$O$ processo de triagem pode ser feito de forma totalmente manual ou automaticamente, ou mesmo semiautomática. A Tabela 1 resume as principais vantagens de desvantagens dos métodos de triagem.

\begin{tabular}{|c|c|c|c|}
\hline & \multicolumn{3}{|c|}{ Métodos de triagem } \\
\hline & $\begin{array}{l}\text { Triagem } \\
\text { manual }\end{array}$ & $\begin{array}{l}\text { Triagem } \\
\text { automática }\end{array}$ & $\begin{array}{l}\text { Triagem se- } \\
\text { miautomática }\end{array}$ \\
\hline Vantagens & $\begin{array}{l}\text { Geração de } \\
\text { muitas vagas } \\
\text { de empregos; } \\
\text { Baixo investi- } \\
\text { mento inicial; } \\
\text { Melhor dis- } \\
\text { tribuição dos } \\
\text { lucros }\end{array}$ & $\begin{array}{l}\text { Capacidade de } \\
\text { separação es- } \\
\text { timada na im- } \\
\text { plantação da } \\
\text { obra; Alta qua- } \\
\text { lidade dos pro- } \\
\text { dutos separa- } \\
\text { dos; Processo } \\
\text { confiável }\end{array}$ & $\begin{array}{l}\text { Impacto so- } \\
\text { cial positivo } \\
\text { na cidade; } \\
\text { Integração de } \\
\text { catadores e } \\
\text { indústria mo- } \\
\text { derna; Geração } \\
\text { de renda } \\
\text { distribuiída }\end{array}$ \\
\hline Desvantagens & $\begin{array}{l}\text { Triagem limi- } \\
\text { tada (depen- } \\
\text { de das pes- } \\
\text { soas); Exige } \\
\text { capacitação } \\
\text { técnica }\end{array}$ & $\begin{array}{l}\text { Diminui a } \\
\text { quantidade de } \\
\text { funcionários; } \\
\text { Geração de ri- } \\
\text { queza mais con- } \\
\text { centrada; Exige } \\
\text { um alto investi- } \\
\text { mento inicial }\end{array}$ & $\begin{array}{l}\text { Exige maior } \\
\text { controle de } \\
\text { funcionários } \\
\text { e sistema de } \\
\text { automação; } \\
\text { Funcionários } \\
\text { devem ser } \\
\text { qualificados }\end{array}$ \\
\hline
\end{tabular}

Tabela 1. Vantagens de desvantagens dos métodos de triagem Fonte: Adaptado de Machado (2013)

De acordo com Machado (2013), a triagem manual é indicada para cidades pequenas onde o volume de produção não justifica uma central automatizada. Já a triagem automática é para cidades grandes onde o volume de produção não possibilita o trabalho manual. A triagem semiautomática, por sua vez, é indicada para cidades médias onde é possível combinar o trabalho com as associações de catadores de lixo e sistemas automatizados.

A triagem automática e semiautomática dispõe principalmente do uso de esteiras, já a triagem manual é feita com o uso de silos e mesa de triagem. A Figura 1 permite comparar as soluções mais adequada a cada situação.

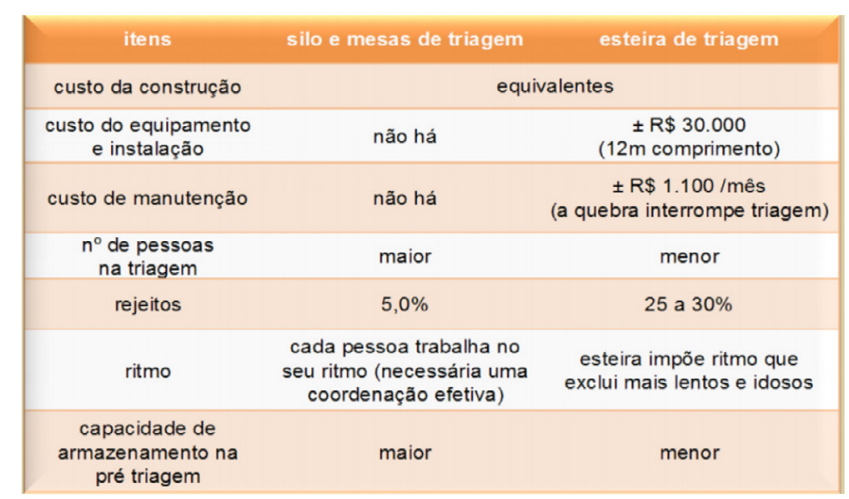

Figura 1. Comparação entre o uso de esteiras e uso de silo e mesas de triagem. Fonte: Brasil (2008)

\section{RESULTADOS E DISCUSSÃO}

A central de triagem proposta para a cidade de Campina Grande-PB é do tipo semiautomática com o uso de esteiras e catadores para auxiliar na segregação dos resíduos. Tal escolha é devido ao fato de Campina Grande ser uma cidade de médio porte, além de haver uma necessidade de inclusão social dos catadores de lixo.

Visando subsidiar a seleção para o aporte de recursos no Programa PAC 2 para resíduos sólidos e apoiar iniciativas de destinação e disposição final de resíduos sólidos urbanos de maneira ambientalmente adequada, o ministério das cidades apresenta diretrizes para o planejamento e execução e centrais de triagem de resíduos Elementos para a Organização da Coleta Seletiva e Projeto dos Galpões de Triagem.

Essa regulamentação define que a licitação é dispensável na contratação da coleta, processamento e comercialização de resíduos sólidos urbanos recicláveis ou reutilizáveis, em áreas com sistema de coleta seletiva de resíduos, efetuados por associações ou cooperativas formadas exclusivamente por pessoas físicas de baixa renda reconhecidas pelo poder público como catadores de materiais recicláveis, com o uso de equipamentos compatíveis com as normas técnicas, ambientais e de saúde pública (PITA, 2011).

Ainda Pita (2011), é preciso a definição de projetos de galpão eficientes, para que seja melhorada a renda dos catadores, além de um processo de seleção de materiais que resulte no baixo índice de rejeito.

Para erguer o galpão da central de triagem, deve ser dada preferência ao uso de materiais pré-fabricados de concreto e metal e sugere a análise da possibilidade de uso de mezanino. O fechamento da alvenaria deve prever segurança a incêndio e a estrutura metálica deve ser externa a ela. Para a abertura da cobertura, recomenda-se ventilação superior cruzada para melhorar a condição térmica do galpão (BRASIL, 2008). 
Para áreas onde está previsto o tráfego de caminhões e demais veículos pesados, o ministério salienta a importância de ter material de maior resistência. Também é preferível que os galpões contenham sistema de energia solar.

Segundo Pita (2011), o galpão deve ser composto de uma área de descarga, silo com área para armazenar um dia e meio a dois dias da coleta diária prevista, uma área para triagem primária e secundária, área para prensagem, uma para estoque dos fardos e expedição com capacidade para armazenar mais ou menos uma semana de cargas fechadas.

D'Almeida e Vilhena (2000) ressaltam que uma usina de triagem e compostagem, quando bem operada, permite diminuição de $50 \%$, em média, do volume de resíduos sólidos que seria destinado aos aterros. Como pode-se perceber, as usinas não conseguem atingir esses patamares de aproveitamento.

As baias intermediárias devem usar estruturas em perfis metálicos sendo que as telas metálicas devem ser de fio grosso com dispositivo de travamento superior e fechamento frontal. A Figura 2 ilustra a forma típica de organização de um galpão.

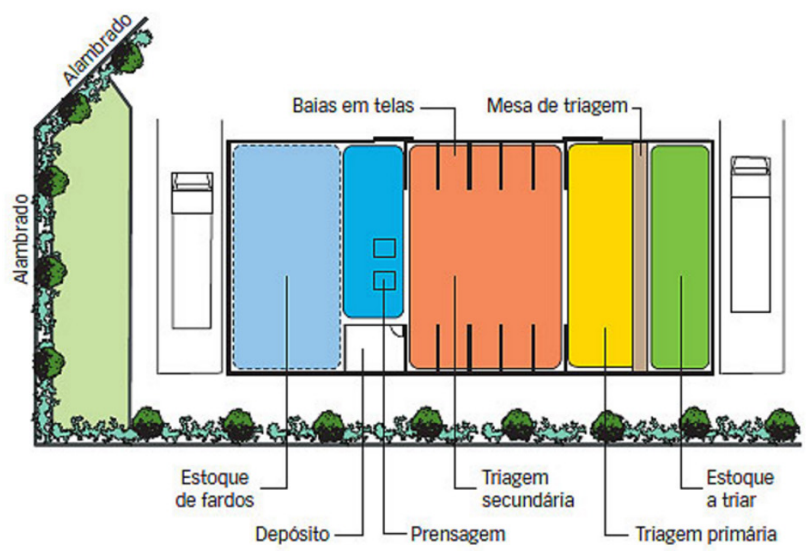

Figura 2. Organização dos espaços do galpão. Fonte: Pita (2011).

A organização da área de triagem é um elemento fundamental que influenciará a eficiência do galpão como um todo. A quantidade de tipos de materiais resultantes da separação deve ser definida de acordo com as características do mercado em cada localidade. É inútil separar materiais que posteriormente terão de ser comercializados como se fosse um único tipo, mas é mais vantajosa a comercialização de materiais mais segregados sempre que possível (BRASIL, 2008).

De acordo com Silva et al.,(2019) ,no caso de as usinas receberem mais resíduos orgânicos, como de podas de árvore, resíduos de restaurante e das Centrais de Abastecimento (CEASA), por exemplo, poderia haver maior qualidade do produto final, tanto pela taxa superior de matéria orgânica desses tipos de fonte, como pela menor contaminação por outros tipos de resíduos, comparada aos RSU domiciliares

A organização da triagem permite a operação de um número maior de pessoas trabalhando no setor de triagem por unidade de área. A Figura 3 mostra uma forma de organização para mesa/esteira linear,o esquema apresentado permite obter 16 tipos diferentes de material, triados e colocados nos tambores ou sacos.

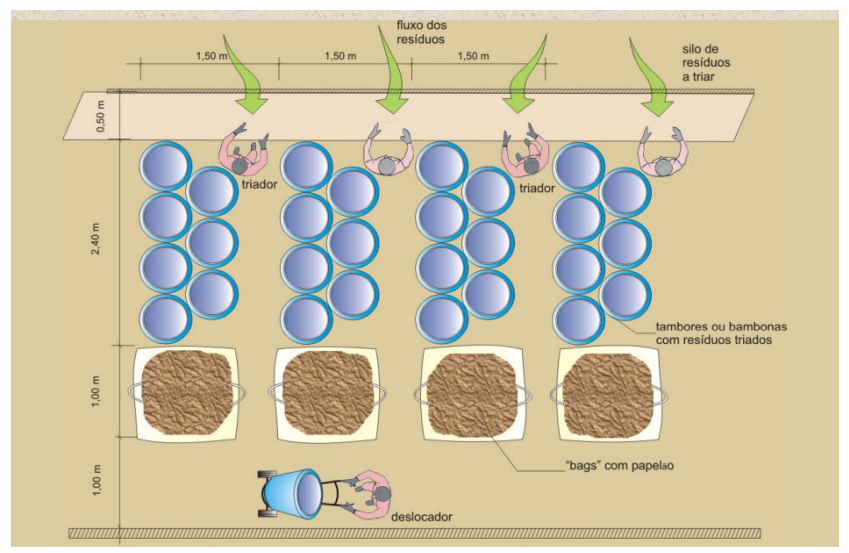

Figura 3. Triagem em mesa linear Fonte: Brasil (2008)

As esteiras por onde passa o resíduo para triagem dos catadores possui velocidade inadequada para catação manual. De acordo com Monteiro et al. (2001) as esteiras de triagem devem ter velocidade entre $0,17 \mathrm{~m} / \mathrm{s}$ e $0,2 \mathrm{~m} / \mathrm{s}$, permitindo um bom desempenho dos trabalhadores que fazem a catação.

De acordo com o Ministério das Cidades (2010), a área do escritório deve ter, no mínimo, $12 \mathrm{~m}^{2}$. Para calcular o número de vasos sanitários e lavatórios, o órgão indica a proporção um para cada 20 usuários. No caso de chuveiros, seria um para cada dez usuários. O boxe mínimo para sanitários deve ser de $1 \mathrm{~m}^{2}$ e a largura mínima para lavatório de 0,6 m. Os armários devem ser individuais com $1,5 \mathrm{~m}^{2}$ por usuário e compartimento duplo com $90 \mathrm{~cm}$ de altura, $30 \mathrm{~cm}$ de largura e $40 \mathrm{~cm}$ de profundidade.

A área por usuário no refeitório deve ser de $1 \mathrm{~m}^{2} \mathrm{com}$ pia, bebedouro, aquecedor de marmitas e fogão, como ilustra a Figura 4. A possibilidade de conversão do refeitório em área de treinamento e reunião deve ser prevista. Para isso, é indicado o uso de mesas móveis (PITA, 2011). 


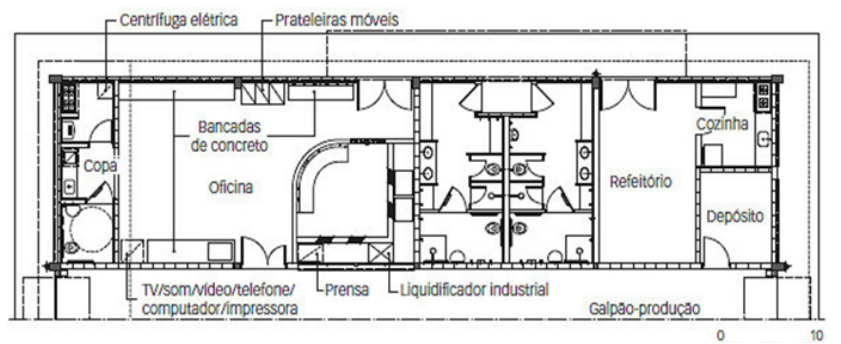

Figura 4. Organização das instalações de apoio. Fonte: Pita (2011)

A Tabela 2 apresenta os equipamentos necessários e seus respectivos preços para um galpão de médio porte, com cerca de $600 \mathrm{~m}^{2}$ edificados.

Os materiais recicláveis promovem o retorno da matéria prima ao setor produtivo, buscando na coleta seletiva uma solução alternativa e ao passo que contribuem para gestão ambiental, impedindo que os materiais recicláveis sejam descartados erroneamente ou aterrados (SANTOS et al.,2020).

\section{CONCLUSÃO}

Uma central de triagem é um empreendimento essencial para a efetivação de um sistema de gerenciamento de resíduos sólidos de uma cidade.

No caso de Campina Grande, a instalação de uma central de triagem seguindo os direcionamentos propostos nesse manual irá viabilizar a etapa de coleta seletiva e reciclagem, fazendo com que o gerenciamento de resíduos da cidade se enquadre no modelo instituído pela PNRS.

Por fim, este artigo busca contribuir como uma referência em termos de critérios a serem analisados no processo de implantação e operação de triagem de resíduos sólidos urbanos.

\begin{tabular}{|c|c|c|c|c|c|c|}
\hline Produto & Quant & Modelo/ característica & Fornecedor 1 & Preço unit (R\$) & Fornecedor 2 & Preço unit \\
\hline Esteira & & $\begin{array}{l}\text { ESR } 1000 \text { e Lemaqui } \\
\text { ESTH8R1000 }\end{array}$ & Kubitz & $\begin{array}{l}23000,00 \\
(E S R 1000)\end{array}$ & Mecalux & $\begin{array}{l}\text { 25400,00 (Lemaqui } \\
\text { ESTH8R1000) }\end{array}$ \\
\hline Prensa enfardadeira & 1 & PAV - 300 e PHV DT 05 & $\begin{array}{l}\text { Ability } \\
\text { Equipamentos }\end{array}$ & $\begin{array}{l}15750,00 \text { (prensa } \\
\text { PAV - 300) }\end{array}$ & $\begin{array}{l}\text { Prensas } \\
\text { Detroit }\end{array}$ & $\begin{array}{l}11300,00 \text { (pren- } \\
\text { sa PHV DT 05) }\end{array}$ \\
\hline Balança & 1 & $\begin{array}{l}\text { Balança mecânica comer- } \\
\text { cial Welmy, } 1000 \mathrm{~kg}, 108 \mathrm{CH}\end{array}$ & Compra fácil & 2499,00 & $\begin{array}{l}\text { Ibirangashop. } \\
\text { com }\end{array}$ & 2499,00 \\
\hline Carrinho plataforma & 1 & $\begin{array}{l}\text { Comprimento: } 1500 \mathrm{~mm} \text {; } \\
\text { Largura: } 800 \mathrm{~mm} \text {; Altura: } \\
\text { 446mm; Capacidade: } 800 \mathrm{~kg}\end{array}$ & Sermap & 1493,17 & $\begin{array}{l}\text { Mercado das } \\
\text { ferramentas }\end{array}$ & 1975,64 \\
\hline $\begin{array}{l}\text { Empilhadeira } \\
\text { simples }\end{array}$ & 1 & LE $1034 \mathrm{C}$ & RR Máquinas & 9334,00 & $\begin{array}{l}\text { Emílhadeira } \\
\text { elétrica }\end{array}$ & 9238,00 \\
\hline
\end{tabular}

Tabela 2. Cotação dos equipamentos a serem utilizados na central de triagem.

Fonte: Brasil (2008)

\section{REFERÊNCIAS}

NBR 10004, ASSOCIAÇÃO BRASILEIRA DE NORMAS TÉCNICAS.: Resíduos sólidos - Classificação. Rio de Janeiro, 2004.

BRASIL, Panorama dos Resíduos Sólidos no Brasil. Resíduos Sólidos. São Paulo: Abrelpe, 2018.

BRASIL. Ministério das cidades. Elementos para a Organização da Coleta Seletiva e Projeto dos Galpões de Triagem, 2008. Disponível:http://www.mma.gov. br/estruturas/srhu_urbano/_publicacao/125_publicacao20012011032243.pdf.

BRASIL. Ministério do Meio Ambiente. Política Nacional de Resíduos Sólidos,2010. Disponível: http://www. mma.gov.br/cidades-sustentaveis/residuos-solidos/ politica-nacional-de-residuos-solidos.

D'ALMEIDA, M.L.O.; VILHENA, A. Lixo Municipal: manual de gerenciamento integrado. $2^{\circ}$ ed. IPT/CEMPRE,370p. São Paulo, 2000.
ELK, A. G. H. P. V. Redução de emissões na disposição final. Segala. Rio de Janeiro: IBAM, 2007.

JACOBI, Pedro Roberto; BESEN, Gina Rizpah. Gestão de resíduos sólidos em São Paulo: desafios da sustentabilidade. Estudos avançados, v. 25, n. 71, p. 135-158, 2011. MACHADO, G. B. Central de Triagem. Portal Resíduos Sólidos. Disponível em: http://www.portalresiduossolidos.com/central-de-triagem/.

MARTINS, Karen Nogueira; CRUZ, C. S.; COUTO, Maria Claudia Lima. Composição de custos de implantação e operação de centrais de valorização de resíduos sólidos urbanos secos. Revista Científica FAESA, v. 12, n. 1, p. 23-30, 2016.

MARTINHO, Maria da Graça Madeira; GONÇALVES, Maria Graça Pereira. Gestão de resíduos. 2000.

MONTEIRO, J. H. P. Manual de Gerenciamento Integrado de Resíduos Sólidos. IBAM, 200p. Rio de Janeiro, 2001. 
OLIVEIRA, S; PASQUAL, A. Gerenciamento de Resíduos Sólidos Urbanos (RSU) em Médias e Pequenas Comunidades. In: XXVI Congreso Interamericano de Ingeniería Sanitaria y Ambiental. Lima - Peru, 1998. SILVA, Fernanda Lemos; CONTRERAS, Francisco. Processo de funcionamento e análise da eficiência das usinas de triagem e tratamento de resíduos sólidos urbanos do Distrito Federal. Anais... IBEAS-Instituto Brasileiro de Estudos Ambientais, Gramado-RS. Disponível em: $<$ https://www. ibeas. org. br/conresol/ conresol2018/XV-028. pdf>. Acesso em, v. 15, n. 08, 2019.

SOARES, Joyce Aristercia Siqueira; PEREIRA, Suellen Silva; CÂNDIDO, Gesinaldo Ataíde. Gestão De Resíduos Sólidos E Percepção Ambiental: Um Estudo com Colaboradores do Campus I da Universidade Estadual da Paraíba. Revista Saúde e Meio Ambiente, v. 4, n. 1, p. 39-54, 2017.

PITA, M. Equipamentos públicos - Centrais de Triagem. Infraestrutura Urbana. Disponível em: http://infraestruturaurbana.pini.com.br/solucoes-tecnicas/10/artigo243539-2.aspx.

SEIDEL, Juliana Matos. Um Problema UrbanoGerenciamento de Resíduos Sólidos e as Mudanças Ambientais Globais. V Encontro Nacional da Anppas, v. 4, p. 1-8, 2010.

SANTOS, Bárbara Daniele; CURI, Rosires Catão; SILVA, Monica Maria Pereira. análise ambiental de empreendimentos de catadores de materiais recicláveis em rede, campina grande, paraíba, brasil. Revista IberoAmericana de Ciências Ambientais, v. 11, n. 5, 2020.

VITOR, A. L.; FALANGO, D.; OLIVEIRA, H. B. D.; LOPES JUNIOR, J. R.; PELLOZO, L. R.; BORGES, L. R. M. F.; PONTELLI, R. C. N.; DALLORA, M. E. L. V. Avaliação de Ferramenta para Gestão de Resíduos em um Hospital Universitário de Nível Terciário. Revista da Faculdade de Medicina de Ribeirão Preto e do Hospital dasClínicas da FMRP,48 (1), 77-86, 2015.

\section{AUTORES}

ORCID: https://orcid.org/0000-0002-7920-4107

VANESSA ROSALES BEZERRA, M.Sc. | Universidade Estadual da Paraíba | PPGCTA - Doutoranda Engenharia Ambiental| Campina Grande, PB. Brasil | Correspondência para: Rua Juvêncio Arruda, S/N - CEP: 58429-600 - Campus Universitário, Bodocongó - Campina Grande - PB | E-mail: rosalesuepb@gmail.com

ORCID: https://orcid.org/0000-0002-1462-5963

LUIS REYES ROSALES MONTERO, Dr. | Universidade Federal de Campina Grande | DEE - Departamento de Engenharia Elétrica | Campina Grande, PB. Brasil | Correspondência para: Rua Aprígio Veloso, 882 - Bairro Universitário Campina Grande - PB - CEP 58429-900 | E-mail: professorluisreyes@hotmail.com

ORCID: https://orcid.org/ 0000-0001-5861-7407

VALDERI DUARTE LEITE, Dr. | Universidade Estadual da Paraíba | PPGCTA - Programa de Pós Graduação em Ciências e Tecnologia Ambiental | Campina Grande, PB. Brasil | Correspondência para: Rua Juvêncio Arruda, S/N - CEP: 58429-600 - Campus Universitário, Bodocongó - Campina Grande - PB | E-mail: mangabeiraleite@hotmail.com

ORCID: https://orcid.org/0000-0001-9583-8671

ADRIANO OLIVEIRA DA SILVA | Universidade Estadual Da Paraíba | PPGCTA - Programa de Pós Graduação em Ciência e Tecnologia Ambiental | Campina Grande, PB. Brasil | Correspondência para: Rua Juvêncio Arruda, S/N - CEP: 58429-600 - Campus Universitário, Bodocongó - Campina Grande-PB | E-mail: adrianoable222@gmail.com

\section{ORCID: https://orcid.org/0000-0002-8921-3576}

YOHHANA JAMILA VILAR BRITO, M.Sc. | Universidade Estadual Da Paraíba | PPGCTA - Programa de Pós Graduação em Ciência e Tecnologia Ambiental | Campina Grande, PB. Brasil | Correspondência para: Rua Juvêncio Arruda, S/N - CEP: 58429-600 - Campus Universitário, Bodocongó Campina Grande - PB | Email: yohhanajvb@gmail.com

\section{ORCID: https://orcid.org/0000-0002-1301-6066}

CARLOS ANTÔNIO PEREIRA DE LIMA, Dr. | Universidade Estadual Da Paraíba | PPGCTA - Programa de Pós Graduação em Ciência e Tecnologia Ambiental | Campina Grande, PB. Brasil | Correspondência para: Rua Juvêncio Arruda, S/N - CEP: 58429-600 - Campus Universitário, Bodocongó Campina Grande - PB | E-mail: caplima@uepb.edu.br 


\section{COMO CITAR ESTE ARTIGO}

BEZERRA, Vanessa Rosales; MONTERO, Luis Reyes Rosales; LEITE, Valderi Duarte; SILVA, Adriano Oliveira da; BRITO, Yohanna Jamila Vilar de; LIMA, Carlos Antônio Pereira de. Proposta De Implantação De Uma Central De Triagem De Resíduos Sólidos Em Campina Grande-PB. MIX Sustentável, [S.I.], v. 7, n. 2, p. 19-26, abr. 2021. ISSN 24473073. Disponível em:<http://www.nexos.ufsc.br/index.php/mixsustentavel>. Acesso em: dia mês. ano. doi:https://doi. org/10.29183/2447-3073.MIX2021.v7.n2.19-26.

DATA DE ENVIO: $13 / 04 / 2020$

DATA DE ACEITE: 02/07/2020 
